\title{
La mise en scène de la violence politique à travers les conférences de presse du FLNC
}

\section{Xavier Crettiez}

\section{(2) OpenEdition}

\section{Journals}

Édition électronique

URL : http://journals.openedition.org/conflits/419

DOI : $10.4000 /$ conflits.419

ISSN : $1777-5345$

Éditeur :

CCLS - Centre d'études sur les conflits lilberté et sécurité, L'Harmattan

Édition imprimée

Date de publication : 15 mai 1993

ISSN : 1157-996X

Référence électronique

Xavier Crettiez, «La mise en scène de la violence politique à travers les conférences de presse du

FLNC », Cultures \& Conflits [En ligne], 09-10 I printemps-été 1993, mis en ligne le 27 janvier 2003, consulté le 30 mars 2021. URL : http://journals.openedition.org/conflits/419 ; DOI : https://doi.org/ $10.4000 /$ conflits. 419

Ce document a été généré automatiquement le 30 mars 2021

Creative Commons License 


\title{
La mise en scène de la violence politique à travers les conférences de presse du FLNC
}

\author{
Xavier Crettiez
}

1 Tenter d'analyser le phénomène des conférences de presse du FLNC, c'est s'introduire dans ce qui fait peut-être la spécificité de la violence politique en Corse : une autolimitation évidente de la violence physique et une sur-représentation - qu'on pourrait qualifier à tort de folklorique - de la violence symbolique théâtralisée. A l'inverse des autres mouvements terroristes à vocation nationale ou indépendantiste, tels que l'ETA, Iparetarak ou l'IRA, le FLNC fait preuve d'une agressivité toute relative. Si les attentats contre des bâtiments publics ou des complexes touristiques sont légion en Corse, le nombre d'assassinats politiques (c'est à dire perpétrés et revendiqués par le FLNC) ${ }^{1}$ est extrêmement faible (16 morts d'hommes entre juillet 1981 et décembre 1990). Dans la même période, c'est par centaines que se comptent les assassinats basques et irlandais.

Cette auto-limitation, qui définirait la spécificité du cas corse, peut trouver ses sources dans la culture insulaire. C'est ainsi que Daniel Hermant écrit: "Faire preuve de l'existence et du sérieux de l'organisation, ou affirmer sa force, peut se faire en Corse avec des coquetteries techniques, et n'est en rien contradictoire avec une grande économie de moyens. Il s'agit bien souvent d'une démonstration spectaculaire, d'une mise en scène, ou d'une sorte de parade sur le sens ethnologique de laquelle on pourrait s'interroger"2. Dans le même ordre d'idée, José Gil ${ }^{3}$ souligne la traditionnelle "crainte de la mort" en Corse où tout crime de sang entraîne des conséquences nombreuses pour ses auteurs. Cette dimension anthropologique d'une peur de la mort et du processus vendettaire qui s'ensuit (sur le mode violence/vengeance), expliquerait de manière contradictoire la violence certaine de la société corse et, en même temps, auto-limitation $\mathrm{du}$ terrorisme insulaire qui préfère aux exécutions sommaires (touchant rarement les corses) des actions plus spectaculaires (plastiquages, conférences de presse) sans conséquences négatives sur la popularité du mouvement. Le phénomène des conférences de presse rejoint cette volonté politique d'auto- 
limitation de la violence. En assurant au mouvement clandestin une popularité certaine auprès d'une frange de la population insulaire ${ }^{4}$ et une publicité d'envergure nationale, les conférences de presse du FLNC réalisent là deux objectifs de tout mouvement terroriste : instiller crainte et respect chez ses victimes potentielles en s'assurant d'un minimum de connivence avec les média, et fournir au mouvement terroriste une légitimité capable d'asseoir politiquement son action militaire.

Ma relation média-terrorisme

3 Force est de constater, au vu des reportages réguliers sur le FLNC, qu'il existe un lien étroit, nous serions tenté de dire une complicité, entre les média télévisés et le mouvement terroriste corse. Le terme de complicité ne doit pas être pris ici dans son sens moral, comme un processus réfléchi et conscient de la part des acteurs dont l'aboutissement mesuré apparaîtrait comme condamnable, mais plutôt comme la convergence de deux logiques, comme la rencontre objective et non délibérée de deux exigences: celle des terroristes du FLNC désireux d'un maximum de publicité que seules les caméras de télévision leur offrent, et celle des média qui, se prévalant de leur droit et devoir d'information, assurent une retransmission aux actions terroristes. Mais cette situation, en apparence "innocente", comporte une ambiguïté qui vicie la relation normale entre ceux qui font l'événement et ceux qui en rendent compte. Théoriquement, ce qui arrive arrive par soi-même, indépendamment des comptes rendus qu'on pourra en donner par la suite: c'est l'événement qui commande l'information. Dans le cas du terrorisme, les circonstances sont différentes en ce sens que l'attentat est justement perpétré pour qu'on en parle : c'est l'information à venir, en fonction de laquelle on bâtit l'événement qui, d'une certaine manière, gouverne l'événement. Le cas des conférences de presse du FLNC est à cet égard probant.

Eléments de "connivence"

4 Le terrorisme du FLNC, comme tout mouvement terroriste contemporain, utilise la publicité sur laquelle il fonde littéralement son action, l'essentiel de l'acte terroriste n'étant pas qu'il s'accomplisse mais que ça se sache. L'activité du FLNC depuis ses plasticages, sa propagande par graffitis, tracts ou symboles et jusqu'à ses conférences de presse clandestines, est destinée toute entière à faire connaître et reconnaître l'organisation terroriste au niveau national. Les mobiles éthiques, historiques ou sociaux du FLNC qui sont, en principe, destinés à justifier moralement l'emploi de la violence cèdent le pas dans le cas des conférences de presse clandestines à des mobiles esthétiques tournés vers la recherche de l'effet. Plus l'action est spectaculaire et innovante, mieux cela vaut: pour les média mais aussi pour les terroristes. En effet, dans la mesure où ceux-ci fournissent aux média un matériel d'une valeur indiscutable, ils se voient attribuer une publicité gratuite de plus en plus utile à leur stratégie.

5 La retransmission télévisée sur les chaines nationales et locales, aux heures de grande écoute, de presque chaque conférence du Front depuis la première, le 5 mai 1976, jusqu'aux plus récentes, montre l'extraordinaire attrait de ce type de manifestation pour les média. Durant ces dix dernières années, l'INA a collecté 31 diffusions de conférences de presse télévisées d'une moyenne de deux à trois minutes, presque toujours diffusées au Journal Télévisé de 20 heures 5 . Cette complicité journalistique nous a été rapportée par de nombreux journalistes qui ont assisté aux conférences du FLNC. P., journaliste d'une grande chaîne nationale désireux de garder l'anonymat, nous avoue être persuadé que, sans la présence des caméras dans l'île, le FLNC se ferait plus discret, et va jusqu'à ajouter : "Les mecs ils font pas une conférence de presse puis 
nous appellent pour y assister, c'est quand on est dans l'île qu'ils décident d'en faire une...". P. parle de l'attaque par le FLNC d'une caravelle sur l'aéroport d'Ajaccio et déclare : "Les mecs n'auraient probablement rien fait si on n'avait pas été dans l'île. Ils voulaient que la presse soit là pour filmer, un mec nous a abordé dans un café en nous disant: soyez dans une heure à l'aéroport, va y avoir du grabuge"6. P. souligne également les relations parfois étroites qui lient les hommes de média au mouvement clandestin: "La première fois qu'on assiste à une conférence de presse c'est impressionnant, mais après tu te calmes, c'est du pipeau. Quand on les connaît un peu, on peut rigoler. J'leur ai dit, car on avait eu une semaine d'enfer avec les nuits bleues ${ }^{7}$ et le coup de l'aéroport: ne nous faites pas de conférences de presse cette nuit"8. P. avoue un peu plus tard connaître certains membres du FLNC, mais n'a jamais vu leur visage : "Les mecs sont toujours masqués, toujours, mais on reconnaît les voix ; genre : eh !, on t'a reconnu Gérard (rires)" 9.

Un autre journaliste régional de FR3 Corse, Bernard Dilasser, souligne, en racontant les contacts qu'il a pu avoir avec les hommes du FLNC lors du transport vers le lieu de la conférence de presse, ce rapport de connivence entre les média et les terroristes. "Ils nous font entrer par la porte latérale et nous font nous coucher immédiatement à l'intérieur du fourgon et, à ce moment là, on est acheminé... mais ils ont des rapports avec la presse qu'ils n'ont peut-être pas avec d'autres personnes, mais avec nous ils ont... c'est pas une complaisance, mais bon... leur action n'a d'impact que si elle est médiatique, donc ils nous ménagent... bon c'est vrai ils sont très cordiaux, ils ont aucune raison de nous bousculer... s'ils nous sollicitent déjà à l'origine, c'est pas ensuite pour nous taper dessus ; mais bon c'est cordial...". Ce même journaliste raconte encore : "c'est vrai qu'on dit souvent FR3 c'est pas FR3, c'est FLN3. Effectivement s'il n'y avait pas les média, le mouvement nationaliste n'aurait pas l'audience qu'il a aujourd'hui... et d'ailleurs c'est nous qui avons pris les coups pour eux souvent aussi... On a fait l'objet en douze ans, moi je suis là depuis les origines, de 54 perquisitions, ce qui est énorme, ce qui s'est jamais vu nulle part (...)"10. Ainsi une certaine connivence entre journalistes et terroristes est reconnue par les acteurs eux-mêmes. Mais les média doivent impérativement se renouveler sous peine de lasser le public. De ce fait, les terroristes doivent eux-mêmes innover pour ne pas se voir délaisser par les média. C'est là le véritable danger que court le terrorisme: celui d'être ignoré ou d'obtenir une couverture journalistique insuffisante. Ce risque de délaissement peut se faire ressentir à deux niveaux. Alors que le terrorisme est destiné à former un contraste marqué avec la routine quotidienne, il peut finir dans certaines circonstances par entrer lui-même dans la routine et par provoquer l'indifférence du public. L'originalité du FLNC est d'avoir innové dans le contenu de ses prestations scéniques : la tenue vestimentaire, la qualité des armes, le positionnement des hommes, leur nombre et le décorum n'ont cessé d'évoluer vers une plus grande spectacularisation. L'objectif est bien entendu de retenir l'oeil du spectateur et réveiller en lui les mythes, stéréotypes et fantasmes en accord avec des aspirations de son époque.

7 De même, l'auto-promotion du FLNC a considérablement évolué au cours des années. Ainsi P. nous déclare-t-il : "Les mecs ont pris en compte l'importance de l'image. Avant, les cassettes qu'ils distribuaient, c'était des cassettes audio, maintenant c'est des cassettes vidéo ; ils ont même un vrai film, c'est des vrai pros" ${ }^{11}$. Dans le même ordre d'idée, Jean Marc Leccia ajoute: "La grande évolution, c'est que c'est nettement beaucoup plus pointu, professionnel qu'avant. Au niveau de la logistique, de l'organisation, ça n'a plus ce coté, je dirai encore une fois... amateur, un peu 
approximatif"12. L'édition en 1991 par le journal à tendance nationaliste Paese d'une cassette vidéo sur le FLNC - L'histoire du FLNC, le FLNC dans l'histoire - véritable film de propagande nationaliste ainsi que l'instauration d'un service minitel ${ }^{13}$ éditant les proclamations du mouvement clandestin et son projet de société, mettant également à disposition des usagers une messagerie, tout cela montre l'importance télévisuelle ou télématique du combat pour l'image que mène le FLNC. Loin de se suffire et de se complaire dans un combat terroriste classique, à l'instar des autres mouvements terroristes nationalistes de type ETA ou IRA, le FLNC n'hésite pas à tout mettre en oeuvre pour s'assurer la publicité la plus large possible. Le terrorisme peut également perdre le bénéfice positif de sa couverture médiatique en évoluant vers une excessive combativité. Ainsi comme l'écrit Philippe Braud: "De même que le spectacle de la guerre est le meilleur antidote au goût de la guerre, le regard qui s'attarde sur les désolations causées disqualifie la violence de rue, et le prestige du terrorisme spectaculaire se trouve sali par la vue des souffrances infligées" ${ }^{14}$. Pour en écarter le risque, la stratégie médiatique du FLNC à travers ses conférences de presse clandestines, repose sur la tentative d'imposition d'une image soft du terrorisme dont la violence se cantonne dans l'ordre du symbolique, évitant la dénonciation de cruauté ou de barbarie et le risque de banalisation de l'horreur qui peut s'en suivre, tout en conservant ce qui fait la force médiatique du terrorisme, une représentation fantasmée d'une violence prohibée en société.

Trois atouts médiatiques des conférences de presse du FLNC

8 Le terrorisme contemporain est devenu pour les média une source de satisfactions professionnelles. Son développement dans les dernières années a conduit des journalistes à devenir des professionnels de la question. Cette évolution répond, bien sûr, à un besoin et à une conjoncture. Mais l'attrait des média pour les terroristes répond également à une logique consciente, et parfois inconsciente, que Pierre Mannoni exprime en ces termes: "Il n'est pas rare de constater en matière d'information sur le terrorisme, un glissement vers un discours hagiographique et apologétique. Tout se passe comme si les média étaient fascinés par ceux qui transgressent les interdits, bousculent les valeurs établies, méprisent le contrôle social et mettent en échec les forces de sécurité et les gouvernements. Aussi utilisent-ils spontanément pour les désigner des formule qui servent d'habitude pour parler des héros et certains comptes-rendus de presse retrouvent spontanément pour les qualifier des expressions et images ordinairement réservées aux personnes légendaires" ${ }^{15}$.

Ceci apparaît particulièrement dans le cas du FLNC. Leur prestation théâtrale, lors des conférences de presse clandestines régulièrement offertes aux média, contient tous les ingrédients nécessaires pour faire vibrer le spectateur. Et la présence des journalistes aux manifestations de ce type présente un triple avantage.

10 Elle permet tout d'abord de surmonter deux contraintes professionnelles majeures. Certaines opérations effectuées par les terroristes peuvent être ressenties par les professionnels de l'information comme des "non-événements". C'est le cas des actions non-immédiatement visibles comme les kidnappings où les média n'ont rien à montrer au public et doivent fournir eux-mêmes une animation, souvent avec beaucoup de difficultés, qui n'apporte que des bribes d'informations, en multipliant les flashes spéciaux afin de maintenir l'intérêt d'un public en attente d'images. Cette multiplication de flashes spéciaux tente, à grand effort d'interviews de spécialistes, de personnalités, de rappels historiques... de camoufler le vide d'information par l'illusion 
d'une situation évolutive en direct. Ce fut particulièrement le cas lors des multiples enlèvements opérés par les Brigades Rouges ou la maffia : "rien ne se passe" mais "tout peut arriver".

11 La seconde contrainte à laquelle peuvent se trouver confronter les média est l'interdiction de filmer l'événement. Lorsque, pour des raisons diplomatiques ou sécuritaires, les gouvernements écartent le droit à l'information et interdisent l'accès à l'événement, celui-ci perd de sa valeur informative et le "spectacle" terroriste fait alors rarement l'actualité.

Les conférences de presse clandestines, parce qu'elles constituent un événement directement enregistré, et retransmissible puisque les journalistes ne subissent pas la censure du pouvoir d'Etat, constituent donc un avantage réel pour les média. L'univers terroriste est par nature un espace secret, difficile d'approche, où la clandestinité et la méfiance à l'égard du monde légaliste sont une raison d'être. Dans cet univers clandestin les repères bougent sans cesse et la lisibilité des événements est faible. Cet obstacle structurel à l'analyse rétro-agit sur l'information; il explique la valorisation, à laquelle nous assistons dans le cas du mouvement clandestin corse, du rôle des individus, quitte à en accentuer la dimension exotique ou pittoresque ${ }^{16}$. "Dans un univers clandestin, les faits liés aux individus offrent un repère fixe" affirme Dominique Wolton ${ }^{17}$. Ceci explique le plaisir avec lequel les journalistes réalisent, comme c'est le cas en Corse, des interviews ou des suivis de conférences de presse dans la clandestinité. La dimension spectaculaire de telles rencontres, rend parfois mal à l'aise le spectateur ou le heurte émotionnellement, mais le journaliste y trouve un point de repère dans un univers incertain où tout se déplace, la preuve qu'il remplit là au moins sa tâche d'informateur.

Enfin, les média s'efforcent de souligner l'aspect grandiloquent des conférences de presse du FLNC (gros plans sur les armes, jeux de lumière...); ils insistent dans les commentaires sur la "clandestinité" de la réunion, le "secret" du rendez-vous, "l'important dispositif de sécurité" mis en oeuvre pour assurer la bonne tenue de la conférence. En se plaçant "au coeur de l'action" et en permettant, à travers l'écran, au spectateur d'assister à une manifestation totalement illégale, condamnée et partiellement dangereuse, le journaliste opère ce que nous pouvons appeler un "processus de construction de l'intimité". Il donne l'impression de révéler les dessous de l'organisation, son armement, ses chefs, ses militants, son repère... et tend à donner au spectateur le sentiment de disposer d'une information complète parce qu'elle est détaillée. En effet la caméra est là ; elle s'infiltre dans l'univers secret du terrorisme. Dès lors, l'information en image est réputée juste, solide et sans contestation possible. Cette infiltration dans l'intimité même de l'illégalité terroriste permet aux journalistes de se poser comme les révélateurs des coulisses du terrorisme corse.

Média et terrorisme entretiennent donc une relation de connivence certaine, maintes fois dénoncées par les autorités étatiques, les victimes des attentats, voire certains spécialistes de la question corse. Le FLNC est encouragé à agir du fait que les média acceptent de faire de son activité clandestine un "événement".

La dimension psycho-sociologique des conférences du FLNC

Pierre Mannoni, auteur d'une thèse en psycho-sociologie sur le terrorisme et ses ressorts publicitaires, définit le terrorisme de la façon suivante: "Le terrorisme correspond à l'emploi systématique d'un ensemble de techniques d'une extrême violence, recourant à des moyens souvent archaïques mais à haute valeur symbolique... 
(Il agit) sur un complexe affectivo-cognitif dominé par les représentations collectives, au moyens de chocs psychologiques, facteurs de tensions anxieuses, dans le but de déterminer chez ses victimes un état psychique morbide spécifique. En bref le terrorisme est un laboratoire de la peur dans lequel on peut voir un instrument de déstabilisation et de manipulation du comportement humain"18.

Si certains auteurs ont poussé trop loin l'étude des dimensions psychologiques des modes d'action terroriste jusqu'à ne voir dans les hommes de l'ombre que des sujets cliniques potentiels, tombant ainsi dans les pièges de la psychologie de l'acteur ${ }^{19}$, il convient de ne pas sous-estimer les pouvoirs émotionnels du terrorisme et l'importance de leur propagation par la presse télévisée. En devenant le relais public du terrorisme, le journaliste offre aux spectateurs une panoplie de l'imaginaire et du rêve qui explique pourquoi la presse télévisée se révèle un support particulièrement efficace à la tactique terroriste.

17 Pour Roger Dufour, derrière le journaliste d'information se glisse continuellement un "journaliste de fantasmes"20. Il ne s'agit pas ici d'accuser les média couvrant le FLNC de désinformation. Au contraire la plupart des journalistes que nous avons rencontrés n'ont à aucun moment semblé faire preuve de légèreté éthique vis à vis d'un thème qu'ils savent difficile à traiter. On insistera seulement sur l'utilisation, par le journaliste, de l'actualité terroriste pour fournir au public des aliments, au besoin de penser le monde comme un spectacle imaginaire où les fantasmes les plus cruels et les plus violents peuvent trouver une place.

18 Or, le terrorisme exploite habilement ce besoin de base de vivre à travers son imaginaire, et les techniques modernes audiovisuelles permettent d'y répondre. Le système qui se construit dans l'interaction entre les différents partenaires de la relation : terroristes - média - public, se fonde sur certaines lois du fonctionnement psychique concernant le rêve éveillé : le besoin de rêve, de dramatisation et de spectaculaire, de division manichéenne du monde entre des héros bipolaires représentant à la fois le bien et le mal, le rebelle à l'Etat et le combattant anticolonialiste, le brigand corse et le poseur de bombes, le "fonctionnaire de la mort" pour reprendre une expression de Michel Wieviorka ${ }^{21}$. En apparence le journaliste est un simple témoin de la société dans laquelle il évolue, il n'est que le chroniqueur neutre des activités qui s'y déroulent. Mais en fait il utilise un objectif (au sens optique) qui révèle, particulièrement lors des conférence de presse du FLNC, des appétits et des peurs, signes des préoccupations sociétales.

19 Lorsque le journaliste filme les conférenciers clandestins, on peut dire qu'il contribue à éveiller la violence collective virtuelle ou ce que Roger Dufour appelle la "pensée criminelle collective" : "Il fournit ce qui constitue le système de regard intérieur qui organise la pensée collective sur la violence et sur le crime, ce que nous appelons la pensée criminelle"22. Diffuser des images de clandestins masqués, armés de M16 et de grenades, immobiles de nuit dans le maquis corse, met en branle des processus intrapsychiques particuliers qui contribuent à éveiller les rêves intérieurs des spectateurs : rêves de violence et d'hérö̈sme ${ }^{23}$. Comme l'écrit Dominique Wolton "le terrorisme réactualise certains fantasmes collectifs et le rôle de la presse est essentiel puisqu'elle montre avec empressement ce que les sociétés modernes refoulent avec application" ${ }^{24}$. Cette idée que les média doivent gérer la symbolique de la mort dans une société qui la nie passe par cet éveil de la "violence collective". L'un des effets les plus frappants des 
reportages sur les conférences de presse du FLNC tient sans doute à cette théâtralisation de la mort offerte au public.

Les terroristes quant à eux, ont besoin de se prouver à eux-mêmes qu'ils ont un certain pouvoir. Ils sont en quête d'affirmation identitaire à la fois personnelle et politique, et cela détermine chez eux un grand appétit pour tout ce qui est susceptible de leur renvoyer une image d'eux-mêmes qui les conforte et les rassure. En ce sens comme ils s'exagèrent probablement le sens et la portée de leur action - étant donné les faibles moyens dont dispose le FLNC pour atteindre l'objectif de "libération nationale" - ils sont à la recherche d'images qui justifient cette vision qu'ils ont d'eux-mêmes. Le travail des média, finalement assez valorisant pour les terroristes, peut-être considéré, pour recourir à une expression behavioriste, comme un renforcement positif. A l'effetécho évoqué jusqu'ici, s'ajoute un effet-miroir qui "piège" les terroristes eux-mêmes, devenus prisonnier du cliché que les média diffusent à leur sujet. Bon gré mal gré, il leur faut alors assumer ce "rôle". C'est souvent assez volontiers d'ailleurs qu'ils entrent dans le jeu: bien que l'image soit parfois négative, elle ne manque pas d'un certain prestige. Le nombre important de "militants FLNC de la dernière heure", présents lors des grandes conférences de presse qu'a connues la Corse, montre la popularité de cette image de terroriste dont le "négativisme social" ne semble pas l'emporter sur la starisation médiatique.

21 Toute une subtile dialectique circule entre les média et les terroristes : tout un jeu de va et vient entre l'image que les uns donnent et l'effort que font les autres pour "coller" à cette image. D'où l'ambiguïté relevée par Yves Michaud des discours journalistiques sur la violence terroriste : "ils voudraient réduire une étrangeté, mais ils contribuent aussi à la produire. En faisant une place dans la représentation du social à ce qui a toujours été défini comme le non-social, ils contribuent à sa mise en circulation" 25 .

Ainsi l'image du FLNC est tellement liée à ces démonstrations de force que sont les conférences de presse, qu'il serait dangereux pour lui de cesser ses opérations médiatiques, au risque de briser sa popularité, sa crédibilité. Le terroriste corse est un homme de média et sa figure cagoulée et armée, retransmise à la télévision, marque l'imaginaire collectif national ; s'en séparer serait pour le FLNC renoncer à son identité. La conférence de presse comme source de légitimité du mouvement terroriste

23 Le phénomène des conférences de presse peut également se comprendre comme une tentative, pour le FLNC, d'accéder à une indispensable légitimité politique. C'est à travers la relation de mimétisme qu'il opère, à travers son rapport à l'histoire et enfin grâce à la présence légitimante des média, que le FLNC se présente comme le garant politique de son peuple.

La relation mimétique du terrorisme

24 "Si on est terroriste c'est presque toujours sous le regard de l'autre" déclare Michel Wieviorka ${ }^{26}$. Le terme même de terroriste à travers tout le "négativisme social" que son sens profond rappelle, est un terme hautement accusatoire. Les pouvoirs publics, relayés en cela par les média, dénoncent les "pratiques terroristes du FLNC", les "terroristes corses", les "poseurs de bombes" etc. Tout reportage télévisé sur le mouvement clandestin est annoncé à l'écran par un petit logo avec l'inscription: "Terrorisme".

25 A l'inverse, les militants FLNC ou les sympathisants plus ou moins avoués ${ }^{27}$ réfutent cette appellation disqualificatrice et lui préfèrent les dénominations de "militants 
indépendantistes", "combattants pour la liberté" etc. On dénonce de la même façon les "méthodes terroristes des pouvoirs publics", "l'Etat terroriste"...

Ainsi Daniel Hermant et Didier Bigo peuvent-ils écrire : "La relation terroriste, loin d'être une relation de violence gagnable sur le terrain militaire, est toujours, en premier lieu, une relation politique visant à remettre en cause la prétention au monopole de la violence légitime. La rivalité de légitimité est donc son enjeu principal (...) : à la composante militaire (ici négligeable), s'ajoute et la détermine, la composante du combat symbolique pour l'accession à la monopolisation du discours légitime, ou tout au moins dans un premier temps, par la décrébilisation du discours des tenants $d u$ pouvoir qui doit être considéré comme arbitraire par les tiers, remettant ainsi à égalité (en position symétrique) les deux adversaires" 28 .

27 La rivalité mimétique que l'on observe dans la dialectique terroriste découle du conflit pour l'accès à la légitimité entre l'organisation clandestine et l'Etat. Remettant en cause son fondement même, à savoir son monopole de la violence physique, le mouvement terroriste lui refuse cette légitimité et va tenter d'instaurer la sienne propre, à même de justifier sa représentativité. Mais c'est dans ce conflit symbolique que la relation mimétique va prendre toute son ampleur. Le mouvement terroriste, pour déchoir les pouvoirs publics, va user des mêmes moyens d'action, du même discours que l'Etat au pouvoir. Daniel Hermant et Didier Bigo écrivent fort justement : "Plus la représentation de la violence se structure, plus elle reduplique à son profit et sans s'en apercevoir, les mécanismes par lesquels l'Etat se justifie. Plus le groupe clandestin insiste sur les différences l'opposant aux gouvernants, plus il les imite dans leur organisation matérielle et tactique"29. De même l'Etat, copiant les méthodes violentes des clandestins, tentera d'appuyer sa lutte contre le terrorisme par la création de corps militaires ou policiers spéciaux, d'organisations secrètes chargées de déstabiliser le mouvement ${ }^{30}$.

Cette analyse de la relation mimétique et du conflit pour imposer sa propre légitimité nous semble à même d'expliquer cette mise en scène opérée par le FLNC dans cette manifestation au titre pompeux - mais significatif du poids symbolique de la démonstration - la conférence de presse. Ce cérémonial n'est pas une invention du mouvement corse mais répond à la volonté affirmée de singer l'Etat et ses instances. Ainsi la position même des conférenciers, les "politiques" assis au premier rang, sans armes, et les "militaires" debouts et armés à l'arrière, n'est pas sans rappeler, à la fois les instances étatiques inscrites dans cette dualité politiques/militaires, mais aussi les grandes cérémonies officielles où, le ou les politiques parlent, assis, tandis que se tiennent à l'arrière les forces de police et le personnel de sécurité ${ }^{31}$. L'arsenal exhibé par le FLNC est impressionnant et montre la modernité technologique du mouvement clandestin. Cependant ces armes sont rarement - voire jamais - utilisées et les terroristes corses se contentent en général d'attentats au plastic. La démonstration de puissance militaire, lors des conférences de presse, est certes destinée à impressionner le public, mais peut également être perçue comme la volonté de concurrencer l'Etat sur son terrain. En s'exhibant comme une véritable petite armée, le FLNC s'instaure comme un possible protecteur de la "nation corse" qu'il veut construire.

29 A travers le rituel dont fait montre le FLNC transparaît cette rivalité mimétique ${ }^{32}$ : les positions des hommes, le sérieux qu'ils adoptent pendant leur prestation, l'usage d'objet à forte connotation symbolique tels que la table de la conférence de presse, les lampes de travail et le drapeau corse. L'utilisation du drapeau notamment est 
caractéristique de la mise en scène du pouvoir. Le drapeau ne dit rien en soi, le symbole national (ou régional) est intéressant en ce qu'il produit "des effets émotionnels intenses chez le sujet et sollicite de lui des réactions irrationnelles" ${ }^{33}$. L'usage répété du drapeau à tête de Maure lors de chaque conférence de presse du FLNC a permis au mouvement ce coup de force symbolique d'appropriation de l'emblème corse à la cause indépendantiste. Il concurrence le drapeau français, symbole en d'autres lieux, de l'unité de la nation.

Dans sa forme même, le discours des allocutions présidentielles est rejoint par celui des clandestins corses. Ainsi le "Vive la France" terminant régulièrement les appels du chef de l'Etat, trouve un écho dans le "Viva populu corsu in lotta" ${ }^{34}$ destiné dans les deux cas à mobiliser les émotions communautaires. "Ce dernier mot est moins voué à achever l'interlocution qu'à la recouvrir d'un signe global qui l'articule à ce dont elle n'était que la manifestation subsidiaire" ${ }^{15}$.

L'histoire comme mode de légitimation de l'action violente

31 A travers son discours politique, le FLNC dépasse les traditionnelles interprétations nationalistes de l'histoire insulaire pour chercher une justification à son action, à la fois dans l'historique local, dans sa propre expérience politico-militaire et dans le "sens de l'histoire" qui semble donner raison aux nationalismes.

32 Le FLNC ne se contente pas de l'autonomie revendiquée par ses précurseurs, mais exige l'indépendance totale et sans conditions, et préconise pour cela la lutte armée contre "l'Etat français colonialiste". Dès lors, le recours à l'histoire par le FLNC se transforme en s'imprégnant du caractère profondément antagoniste entre l'Etat français et la Corse, ce qui permet au mouvement terroriste de légitimer son action militaire violente. Plus profondément le FLNC, parce qu'il perçoit l'histoire de la Corse comme une histoire de violence imposée par l'envahisseur, s'érige en descendant légitime d'un passé belliqueux ; il fait l'histoire en même temps qu'il en subit le cours.

33 La réalité présente de la Corse doit selon lui être analysée en référence à l'occupation qui débute avec la défaite du "peuple corse" à Ponte Novu. Depuis cette bataille perdue dans le sang, le FLNC montre, dans son discours historique, que le "peuple corse" oppose une résistance farouche à l'occupation. Résistance qui prend la forme d'une guérilla et d'une lutte armée. "Le mouvement de libération nationale est passé d'une première phase, celle du réveil culturel identitaire caractéristique des années $60-75$, à la nouvelle résistance animée en particulier par notre organisation devenue dépositaire et garante de la conscience collective du peuple corse" ${ }^{136}$.

34 Cette évolution, marquée dans le discours, traduit deux choses. Tout d'abord on s'aperçoit avec Michel Verret, que "tout conflit d'organisation est conflit de mémoires d'organisation" ${ }^{137}$. La date d'entrée en résistance, la forme de cette résistance sont autant d'enjeux politiques légitimateurs de la représentativité du "peuple corse" dont peuvent se prévaloir les organisations nationalistes. Le FLNC s'approprie la légitimité de cette lutte en s'autoproclamant la "nouvelle résistance" du peuple, alors que ses prédécesseurs n'avaient fait qu'éveiller ce même peuple à la conscience nationale.

35 Non content de se servir de l'histoire, le FLNC se présente comme faisant l'histoire. Il suffit pour s'en convaincre de regarder la cassette vidéo, publiée par le journal Paese, sur le FLNC, avec ce titre choc: L'histoire du FLNC, le FLNC dans l'Histoire : 15 ans de lutte, ainsi qu'un commentaire glorifiant "les militants de la liberté". Le FLNC s'inscrit donc dans la mémoire historique du "peuple corse". Chacune de ses interventions télévisées lors de conférences clandestines qu'il donne, lui permet de se situer ou de se 
resituer par rapport à ses précédentes interventions ou à ses propres actions militaires: "notre conférence de presse se situe dans le prolongement de ces quinze années de lutte (...). Nous nous trouvons à quelques centaines de mètres de l'endroit où fut donnée notre précédente conférence de presse du mois d'août dernier..." 38.

Le FLNC s'inscrit également dans une logique plus large, débordant le cadre insulaire, en faisant référence à sa filiation idéologique avec le mouvement des nationalités des pays de l'est de l'Europe ; mouvements qui, par leur force et leur vigueur, ont donné l'impression "d'accélérer" le cours de l'histoire. C'est ainsi que le mouvement clandestin parle des "changements intervenus récemment en Europe par le réveil des nationalités arbitrairement privées d'existence par des système politiques oppresseurs (qui) interpellent tous ceux qui, de par le monde, luttent pour leur souveraineté. Ces changements concernent directement la Corse car notre pays doit prendre sa place dans ce vaste mouvement d'autodétermination" ${ }^{39}$.

La réactualisation du mythe de la Corse indépendante est nécessaire au FLNC pour réveiller le peuple à la conscience nationale et le pousser à participer à la lutte armée contre l'envahisseur français. Elle se poursuit dans le rituel des conférences de presse. La violence extrême des images des conférenciers, armés de fusils M16, grenades à la ceinture et revêtus de treillis militaires, est une vision quelque peu surréaliste et a un effet psychologique très fort sur le spectateur. Le message est clair : l'histoire de la Corse moderne est née dans la violence avec la défaite de Ponte Novu, la réactualisation de cette violence replace la Corse dans son histoire. En d'autres termes, la violence potentielle du Front doit être entendue et perçue par le "peuple corse" comme une invitation à rejoindre son histoire mythique, celle de la lutte contre l'envahisseur pour l'accès à la souveraineté.

Dans cette même optique, le FLNC, dont les apparitions télévisées se font toujours dans le maquis, lieu symbolique de la rébellion corse, reprend à son compte cette longue tradition locale du "banditisme". Les puissants bandits des montagnes avaient

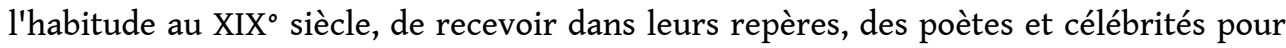
expliquer leurs actions ${ }^{40}$.

La force légitimatrice des média

La spécificité du terrorisme corse, auto-limitation de la violence, s'explique par cette quête d'une légitimité médiatique, conférant au discours du FLNC une autorité légitimant ses prétentions à se dire représentant du "peuple corse".

Les conférences de presse clandestines constituent un rituel d'investiture où le FLNC, intronisé par les média, se trouve consacré et reconnu comme une organisation nationaliste ayant une influence certaine. La conférence de presse apparait comme un "coup de force symbolique" (Pierre Bourdieu) par lequel le FLNC fixe et fige des hiérarchies et s'impose dans la "lutte pour l'imposition de la vision légitime" du combat autonomiste et indépendantiste. Le FLNC, via son discours, son appropriation et sa démonstration d'une violence théâtralisée, entend apparaître comme la branche dominante de la mouvance nationaliste, et le grand porteur de la légitimité populaire ${ }^{41}$. Mais le rituel terroriste ne fonctionne que si la croyance dont il est l'objet est partagée. Or cette croyance en l'importance du mouvement terroriste, en sa détermination, en sa ligne politique, en ses moyens d'action militaire est permise grâce aux journalistes présents sur les lieux qui offrent aux terroristes une reconnaissance publique télévisuelle. 
41 Soulignons une fois encore le caractère officiel du terme de conférence de presse et sa diffusion dans les média, en ce qui concerne le FLNC, qui donne sens à sa quête de légitimitée ${ }^{42}$. Les média fonctionnent ici comme le skeptron de Homère cité par Bourdieu: "l'abondance des micro, des caméras, des journalistes, des photographes ( que l'on voit régulièrement dans les films sur le FLNC, agenouillés face aux terroristes, leur tendant micro et enregistreurs) est, à la manière du skeptron homérique, la manifestation visible de l'audience accordée à l'orateur, de son crédit, de l'importance sociale de ses actes et de ses paroles. La photographie (ou le film) qui, en enregistrant, éternise, a pour effet, ici comme ailleurs, de solenniser les actes exemplaires du rituel politique. Il s'ensuit que l'intervention de cet instrument de perception et d'objectivation désigne les situations où les hommes politiques sont en représentation, agissent pour être vus agissant, donnent la représentation du bon représentant"43.

Dès lors, bénéficiant de ce fort potentiel légitimatoire accordé par la présence des caméras, le discours du Front acquiert une plus grande puissance performative. Les notions de "peuple corse", d'indépendance nationale, de langue corse gagnent en autorité du fait de l'intronisation du porte-parole faisant désormais autorité.

La spécificité du terrorisme corse réside incontestablement dans l'auto-limitation de la violence politique à travers une théâtralisation de la puissance militaire du FLNC. Cet exceptionnalisme insulaire a pu être expliqué sous un angle culturaliste visant à souligner l'angoisse d'une population vis-à-vis de la mort et de ses suites vendettaires. Nous avons voulu adopter une perspective plus sociologique et mettre en avant l'aspect stratégique d'une telle politique. A travers les relations étroites avec le monde des média et, en particulier, la télévision en quête de sensationnalisme, le FLNC parvient à agir sur les populations et à faire reconnaître sa détermination militaire. Mais c'est surtout dans son opposition symbolique à l'Etat national, dans son discours historisant, et grâce à la légitimité que lui confèrent les média, que le mouvement clandestin corse peut s'instaurer en représentant d'un "peuple" et prétendre à une légitimité politique. Le recours à une violence aveugle et plus classique en matière de terrorisme, desservirait le mouvement clandestin en lui ôtant à la fois sa "complicité" avec le champ médiatique et ses prétentions à la "respectabilité" politique qu'il atteint partiellement grâce à lui.

\section{NOTES}

1. Nous excluons ici la vendetta privée ou les crimes mafieux.

2. D. Hermant, Le terrorisme en Europe à l'horizon 92, rapport IHESI, FNSP, 1992, p. 29. Nous tenons à remercier Catherine Gorgeon de l'IHESI qui nous a permis la lecture de ce rapport ainsi que L'Europe de la sécurité intérieure, D. Bigo et R. Leveau, FNSP, 1992. Merci également à FR3 Ajaccio pour le libre accès à ses sources audiovisuelles.

3. Gil (J.), La Corse entre la liberté et la terreur : étude sur la dynamique des systèmes politiques corses, La Différence, 1991. 
4. La diffusion dans la société corse, sous forme de pendentif ou d'autocollant, de l'image du tireur du FLNC mitraillette sur l'épaule, est un indice de la popularité de ces "héros" de l'ombre.

5. Cet article repose sur l'analyse de 12 de ces conférences de presse clandestines, qui nous ont semblé significatives de la stratégie médiatique du FLNC. Elles sont toutes disponibles à l'INA. Il s'agit des conférences de presse des 27/02/1982 - 04/10/1983 19/10/1987 - 12/04/1988 - 30/09/1988 - 30/12/1989 - 15/01/1990 - 06/08/1990 20/09/1990 - 26/11/1990 - 08/01/1991.

6. Entretien avec P., en date du 10 mars 1992.

7. On nomme ainsi les nuits explosives en Corse pendant lesquelles des dizaines d'attentats ont lieu.

8. Entretien avec P., en date du 10 mars 1992.

9. Ibidem.

10. Entretien avec B. Dilasser, en date du 15 avril 1992.

11. Entretien avec P. en date du 10 mars 1992.

12. Entretien avec J.M. Leccia, journaliste à FR3 Ajaccio, en date du 16 avril 1992.

13. 3615 code Paese ou 3615 code Ribombu service FLN. Son existence peut paraître étonnante s'agissant d'un mouvement ouvertement qualifié de terroriste et dissout par les autorités étatiques.

14. Braud (P.), Le jardin des délices démocratiques, FNSP, 1991, p. 152.

15. Mannoni (P.), Un laboratoire de la peur, terrorisme et média, éd. Hommes et perspectives, 1992, p. 160.

16. $P$. confirme ainsi cette hypothèse : "Certains voudraient que je fasse du folklore sur la Corse (il me montre les bureaux de la rédaction), ça leur plaît le folklore (...) mais y'en a marre des images sur la Corse : c'est des fainéants, des violents etc."

17. Dominique Wolton et Michel Wieviorka, Terrorisme à la Une, Paris, Gallimard, 1987, p. 94.

18. Mannoni (P.), op. cit., pp. 15.16 .

19. Voir, à ce sujet, certains points de vue du livre de Friedrich Hacker, Terreur et terrorisme, Flammarion, 1976.

20. Dufour (R.), "Les ressorts psychologiques de l'efficacité publicitaire du terrorisme", Etudes Polémologiques, $1^{\circ}$ trim. 1986.

21. Wieviorka (M.), Société et terrorisme, Paris, Fayard, 1988.

22. Dufour (R.), op. cit., p. 43.

23. Ainsi B. Dilasser déclarait: "Le responsable du S.O. nous disait : vous pouvez faire ça, vous pouvez pas faire ça etc., en plus manifestement, ils ont des pierres dans la bouche, des trucs pour qu'on arrive pas à reconnaître leurs voix, comme dans les films, c'est un décorum qu'est volontaire et c'est vrai qu' c'est impressionnant", entretien du 15 avril 1992.

24. Dominique Wolton et Michel Wieviorka, Terrorisme à la Une, Paris, Gallimard, 1987, p. 115.

25. Michaud (Y.), Violence et politique, Gallimard, 1978, p. 112.

26. Wieviorka (M.), Société et terrorisme, Paris, Fayard, 1988, p. 15.

27. P. nous déclarait lors de notre entretien : "En tout cas, jamais je n'appellerai terroristes ces types là, pour moi c'est pas des terroristes."

28. Hermant (D.) et Bigo (D.), "Les organisations clandestines en France", Etudes Polémologiques, $\mathrm{n}^{\circ} 3,1988$, p. 55.

29. Ibidem, p. 60. 
30. La dénonciation par les nationalistes corses, dont le FLNC, des méthodes d'action de l'Etat français, et en particulier de l'envoi de "barbouzes" destinées à infiltrer et "supprimer" le mouvement clandestin, est significatif de cette mimétique de la violence.

31. C'est ainsi que Jean Marc Leccia, journaliste à FR3 Corse, nous décrivant les conférences auxquelles il a assistées, met l'accent sur ce mimétisme : "On sent la présence d'une espèce de logistique de télécommunication, ça part du talkie-walkie tout simple jusqu'au scanner pour ne pas se faire repérer... comme les flics (...). On sent un progrès même dans l'habillement, $\mathrm{y}$ a une espèce de tenue, de salopette satinée type GIGN..."

32. René Girard écrit : "C'est souvent au moment du paroxysme, juste avant le sacrifice, que les participants revêtent leurs masques, ceux là au moins qui jouent dans la cérémonie un rôle essentiel (...). Les fidèles disparaissent ensuite derrière leurs masques pour se métamorphoser en doubles monstrueux" in La violence et le sacré, Paris, Grasset, 1972, pp. 233-234

33. Michaud (Y.), Violence et politique, Gallimard, 1978, p. 68.

34. Voir le discours de la conférence de Borgu du 25 novembre 1990.

35. Hélias (Y.), La symbolique du pouvoir d'Etat dans les allocutions télévisées des présidents de la république française, Thèse de troisième cycle, université de Rennes, 1983, p. 170.

36. Projet de société "Fronte" in 3615 code Paese.

37. Verret (M.), "Mémoire ouvrière, mémoire communiste", Revue Française de Science Politique, vol. 34, n³, juin 1984, p. 421.

38. Conférence de Borgu du 25 novembre 1990, p. 1.

39. Conférence de presse du 30 décembre 1991, FR3 Ajaccio.

40. Selon Jean Marc Leccia, entretien du 16 avril 1992.

41. Les dissensions visibles au sein du mouvement entre un canal historique, un canal habituel et, épisodiquement, le groupe R. doivent être comprises de la même manière. La rivalité entre ces groupes à coup de conférences de presse clandestines de plus en plus nombreuses et sophistiquées, traduit la lutte en cours pour l'imposition d'une vision légitime du combat indépendantiste. Les termes même de "canal habituel" ou "historique" montrent cette quête de légitimité de l'action politique ; légitimité fondée sur l'histoire du mouvement et l'histoire de la Corse, mémoire vivante et mémoire morte.

42. Il est significatif à cet égard de voir que les journalistes remettent rarement en cause ce terme de "conférence de presse", et même lorsqu'ils soulignent l'aspect dirigiste des démonstrations du FLNC, ils continuent d'user de cette appellation gratifiante et légitimante.

43. Bourdieu (P.), "Décrire et prescrire. Notes sur les conditions de possibilité et les limites de l'efficacité politique", Actes de la Recherche en Sciences Sociales, $n^{\circ} 38$, mai 1981 , p. 15. Cette hypothèse tendant à donner aux journalistes un rôle de tremplin à la légitimité d'un mouvement terroriste rejoint celle de Dominique Wolton tout en s'y opposant dans le fond. Pour l'auteur de Terrorisme à la Une, "non seulement les média ne font pas la publicité du terrorisme mais ils contribuent au contraire à l'affaiblir dans la mesure où ils lui ouvrent une porte par laquelle celui-ci peut quitter la voie de la lutte armée pour entrer dans celle de la politique". Cette vision de la presse comme source de pacification d'un mouvement clandestin nous paraît exagérée tant les journalistes assurent, aux terroristes corses, justifications et légitimités à leur violence. 


\section{RÉSUMÉS}

Le FLNC occupe incontestablement le devant de la scène du terrorisme hexagonal. Pourtant, en dépit de sa longévité historique, de sa capacité militaire et de son implantation organisationnelle en Corse, il se démarque des autres mouvements terroristes à vocation nationale et indépendantiste tels que l'ETA ou l'IRA, par un usage limité de la violence des armes. C'est cette autolimitation de la violence physique - inhabituelle dans le champ terroriste - qui surprend l'observateur. Parallèlement, la théâtralisation de la violence, à travers le phénomène des conférences de presse clandestines, est devenue une forme d'identité propre au FLNC. Simple coquetterie technique, fruit d'une tradition historique ou, plus vraisemblablement, résultat d'une stratégie des " acteurs " la mise en scène guerrière de la contestation nationalitaire oriente la violence sur un espace symbolique, contribuant ce faisant à son autolimitation. Celle-ci apparaît donc comme le résultat des relations étroites que le mouvement clandestin entretient avec les média, lui assurant une présence militaire au niveau national et une tribune politique où affirmer la " légitimité " de sa prétention à représenter le " peuple corse ".

The FLNC (Corsican Liberation Front) undoubtedly holds a central position in the terrorist scene of the French territory. Despite its age, its military and organisational strength in Corsica, it varies from other terrorist movements, such as ETA and the IRA, to the extent that it has little recourse to arms. This self-restraint - unusual in terrorist circles - surprises the observer. However, the dramatisation of violence through secret press conferences has become a distinguishing feature of the FLNC. Whether mere affectation, historical tradition, or, more likely, a carefully prepared stage-set, this technique leads to the creation of a symbolic scene of confrontation permitting self-restraint. Through its close contact with the media, the FLNC achieves a " military presence " at national level, a political platform from which to proclaim its lawful representation of the " Corsican people ".

\section{INDEX}

Index géographique : France

Mots-clés : guérillas et organisations clandestines, séparatismes/ autonomismes, violence, visuel

Thèmes : FLNC 